\title{
Quantum Walks in a Superconducting Quantum Computer
}

\author{
Jaime Santos $^{1,2}$, Bruno Chagas ${ }^{3,4}$, Rodrigo Chaves ${ }^{5}$ \\ ${ }^{1}$ Universidade de Aveiro, Aveiro, Portugal \\ ${ }^{2}$ INESC TEC, Braga, Portugal \\ ${ }^{3}$ Irish Centre for High-End Computing, Dublin, Ireland \\ ${ }^{4}$ National University of Ireland, Galway, Ireland \\ ${ }^{5}$ Universidade Federal de Minas Gerais, Brazil
}

jaimepereirasantos123@gmail.com, bruno.chagas@ichec.ie, rodrigoogchaves@gmail.com

\begin{abstract}
Quantum Walks are among the most widely used techniques with which we can construct new quantum algorithms. This paper aims to outline how to construct a circuit for the continuous-time quantum walk (CTQW) over circulant graphs using the Quantum Fourier Transform (QFT) due to the spectral properties of those graphs. Furthermore, we examine how the Approximate Quantum Fourier Transform (AQFT) allows us to shorten the size of the circuit by reducing the number of controlled rotation gates. The contributions of this paper consist of the development of a general circuit implementation of the $C T Q W$ for an important class of graphs that does not scale up with time, and the study of the cases where the AQFT decreases the error by controlling the approximation. Finally, we provide a statistical analysis for several circulant graphs, running experiments in a IBM's superconducting quantum computer, and we also explore the pretty good state transfer (PGST) for some graphs.
\end{abstract}

\section{Introduction}

Quantum walks provide a versatile framework in which we can develop new quantum algorithms for problems such as searching [Childs and Goldstone 2004] and element distinctness [Ambainis 2008]. This technique has been studied in the context of transport properties such as perfect state transfer [Coutinho 2014] and mixing time [Chakraborty et al. 2020]. The coined quantum walk model was implemented using the topological formulation in a cycle graph [Balu et al. 2018], and by using Quantum Fourier Transform (QFT) as the main tool in the construction of the circuit [Shakeel 2020]. One of the major drawbacks of the coined quantum walk model is that it requires an extra space for the coin, and for some graphs the usage of a Noisy Intermediate-Scale Quantum (NISQ) computer can be impractical. The staggered quantum walk model, which does not require a coin space, was implemented in the cycle and two-dimensional lattice [Acasiete et al. 2020]. Those previous works were limited to the physical realization of discretetime quantum walks, and there's still a need for a larger class of graphs which require a minimum number of qubits, while having low circuit depth.

In the literature we find a physical realization of continuous-time quantum walk (CTQW) in a photonic quantum processor using circulant graphs, but this work is limited

This work is financed by the ERDF - European Regional Development Fund through the Operational Programme for Competitiveness and Internationalisation - COMPETE 2020 Programme and by National Funds through the Portuguese funding agency, FCT - Fundação para a Ciência e a Tecnologia, within project POCI-01-0145-FEDER-030947. 
to a graph with four nodes [Qiang et al. 2016]. Our paper aims to revisit this implementation in a superconducting quantum computer, constructing our quantum circuits with the AQFT, so as to evaluate the error rate by approximating the circuit. We consider the case of 2, 3 and 4 qubits, and we perform a statistical analysis for a variety of circulant graphs.

This paper is organized as follows. Section 2 gives a brief description of CTQWs, describing the circulant graphs and its spectral properties, and the quantum circuit for the AQFT. Section 3 presents our results, performing experiments for several graphs, varying the number of qubits and analysing the effect of AQFT on state fidelity. Finally, we conclude our work in section 4 giving some remarks and insights for further improvements.

\section{Theoretical Background}

The development of new quantum algorithms can be achieved by the manipulation of the Schrödinger equation, which governs the evolution of quantum mechanical systems. Given a quantum state $|\psi(x, t)\rangle$, which belongs to a Hilbert space $\mathcal{H}$, and the Hamiltonian $H$ which encodes the dynamics of an isolated quantum system, we can write

$$
i \hbar \frac{\partial|\psi(t)\rangle}{\partial t}=H|\psi(t)\rangle,
$$

where $\hbar=1$, and its evolution occurs in a $N$-dimensional space. Throughout this work we consider the time-independent case of $H$, and this allows us to find a solution for (1)

$$
|\psi(t)\rangle=U|\psi(0)\rangle=e^{-i H t}|\psi(0)\rangle,
$$

where $H$ is a Hermitian operator considering a closed system.

Given a graph $G(V, E)$ and its underlying adjacency matrix $A$, where $V, E$ are sets of vertices and edges, respectively, we can describe a CTQW by the equation

$$
|\psi(t)\rangle=e^{-i A t}|\psi(0)\rangle
$$

where $A$ is assuming the role of the Hamiltonian [Farhi and Gutmann 1998]. Throughout this work, the circulant class of graphs will be considered. This kind of graph is defined by a circulant matrix, such that

$$
A=\left[\begin{array}{ccccc}
c_{0} & c_{N-1} & \cdots & c_{2} & c_{1} \\
c_{1} & c_{0} & c_{N-1} & & c_{2} \\
\vdots & c_{1} & c_{0} & \ddots & \vdots \\
c_{N-2} & & \ddots & \ddots & c_{N-1} \\
c_{N-1} & c_{N-2} & \cdots & c_{1} & c_{0}
\end{array}\right] .
$$

Considering a simple graph, the adjacency matrix in (4) should have some constraints: firstly, $c_{0}=0$ by the absence of self-loops; secondly, the matrix must be symmetric, meaning that $c_{n-j}=c_{j}$.

In order to construct the quantum circuit, notice that the circulant matrix is diagonalizable by the Fourier transform, such that the eigenvalues are

$$
\lambda_{p}=c_{0}+\sum_{q=1}^{N} c_{N-q} \omega^{p q} .
$$


where $\omega=e^{2 i \pi / N}$. We can then construct an operator which diagonalizes the circulant graph and we can define the unitary operator for the CTQW as

$$
U=F^{\dagger} e^{i \Lambda t} F
$$

where $F$ will be the QFT, and

$$
e^{i \Lambda t}=\sum_{j} e^{i \lambda_{j} t}|j\rangle\langle j|
$$

is a diagonal operator which is implemented using multiplexors [Shende et al. 2006, Bullock and Markov 2004]. Finally, we've implemented the approximate quantum Fourier transform (AQFT), which is similar to the Quantum Fourier Transform, but the least significant controlled rotations are discarded, as depicted in figure 1, considering $m \leq n-1$.

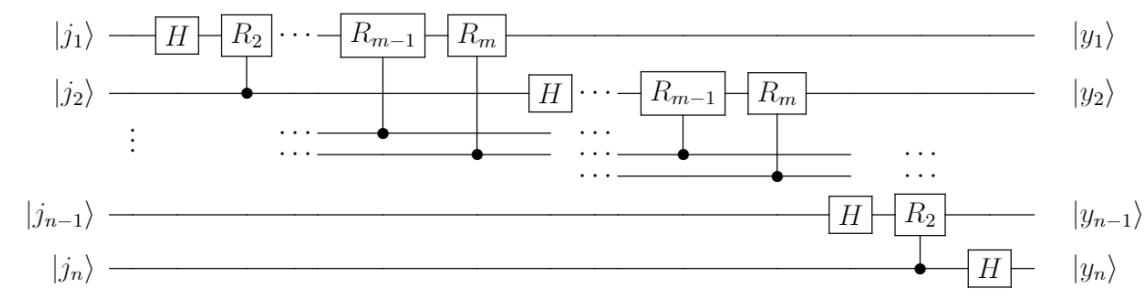

Figure 1. Circuit for the approximate quantum Fourier transform.

\section{Simulating on a Quantum Computer}

In this section we present the methodology used to implement the quantum circuits, and our findings with respective analysis. Firstly, we construct our circuits, as is shown in figure 2 .

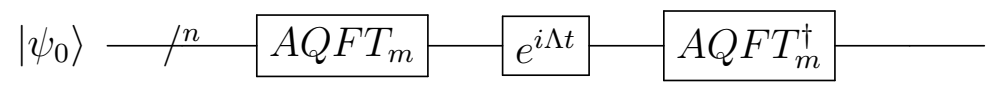

Figure 2. Circuit for the continuous-time quantum walk.

The Qiskit package is then used to submit our jobs to IBM's Toronto backend. In order to construct this circuit, we use two main functions provided by this package. The first one is the $Q F T$, that takes in the number of qubits, $n$, and approximation degree. The second function is named diagonal, which allows to construct circuits for general diagonal operators using multiplexors [Shende et al. 2006, Bullock and Markov 2004].

Each experiment is performed 10 times, with 3000 shots each, in order to extract substantial statistical data, using the confidence interval of $95 \%$. We calculate the average fidelity between the ideal distribution $p(x, t)$ and the experimental $q(x, t)$ using the formula

$$
F(p, q)=\frac{1}{10} \sum_{i=1}^{10} \sum_{x=0}^{N-1} \sqrt{p(x, t) q(x, t)}
$$

where $x$ is the vertex and $t$ time, so we can compare to previous work [Qiang et al. 2016]. 
We selected a considerable number of graphs, as is shown in figure 3 , to observe how the effects of the AQFT differ. For instance, the graph $G_{1}$ is generated by setting $c_{1}$ and $c_{n-1}$ to 1 , and the rest of the elements to 0 , and this corresponds to a cycle graph. Therefore, $G_{k}$ will have elements $c_{k}, \ldots, c_{1}=1$ and $c_{n-k}, \ldots, c_{n-1}=1$. For instance, graph $G_{2}$ with $N=8$ can be described by column vector $[0,1,1,0,0,0,1,1]^{T}$. In this way, we can systematically construct circulant graphs varying from sparse to dense.

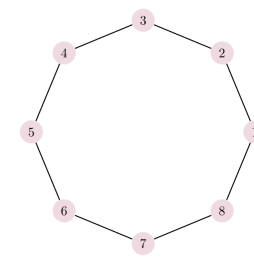

(a) $G_{1}$

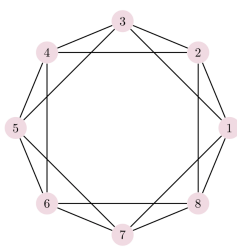

(b) $G_{2}$

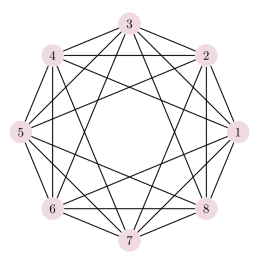

(c) $G_{3}$

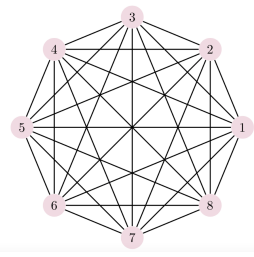

(d) $G_{4}$

Figure 3. Circulant graphs $G_{k}$ for $N=8$ elements.

Figure 4 shows the probability distributions for the CTQW on the $G_{2}$ graph, for $N=8$ elements. The blue bar represents the quantum walk on the QASM simulator, which is not a real quantum computer but an ideal simulation which is not affected by noise. The remaining bar plots were obtained by running the circuit on the Toronto backend, where we can see the error introduced by noise.

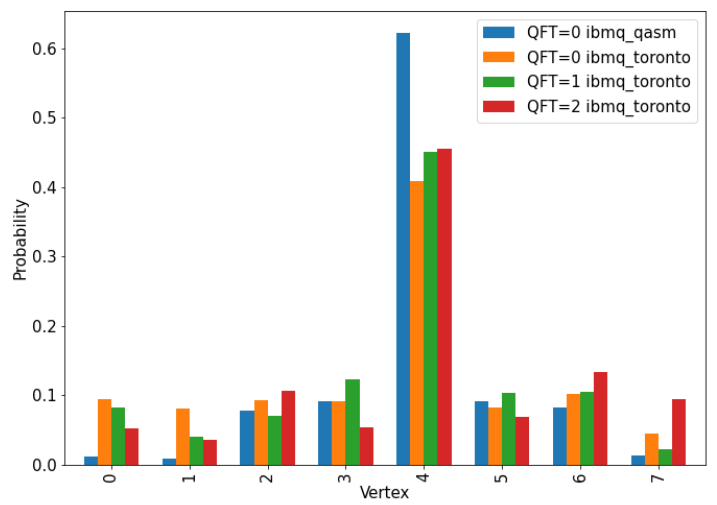

Figure 4. Continuous-time quantum walk for $\mathrm{N}=8$ elements, with time $=1$ and initial condition $|\psi(0)\rangle=|4\rangle$.

For the smaller $N=4$ case, two non-isomorphic circulant graphs are possible to construct. $G_{1}$ corresponds to the cycle graph, and $G_{2}$ to the complete graph. Table 1 shows the achieved average fidelities, which were calculated with (8). Our result for the complete graph slightly outperforms Qiang's et al work [Qiang et al. 2016], where they obtained a fidelity of $0.967 \pm 0.003$.

\begin{tabular}{|l|l|l|}
\hline $\mathrm{m} \backslash G$ & $G_{1}$ & $G_{2}$ \\
\hline 0 & $0.98 \pm 0.01$ & $0.99 \pm 0.01$ \\
\hline 1 & $0.98 \pm 0.02$ & $0.993 \pm 0.006$ \\
\hline
\end{tabular}

Table 1. Fidelity of quantum state with $\mathrm{N}=4$, backend Toronto, and $\mathrm{t}=1$.

In the $N=8$ case, table 2 shows that state fidelity is greater as graph connectivity increases, and as $m$ increases. This is due to the fact that a greater $m$ implies a smaller circuit, due to the approximation, which means it will be less affected by decoherence. 


\begin{tabular}{|l|l|l|l|l|}
\hline $\mathrm{m} \backslash G$ & $G_{1}$ & $G_{2}$ & $G_{3}$ & $G_{4}$ \\
\hline 0 & $0.80 \pm 0.01$ & $0.92 \pm 0.02$ & $0.968 \pm 0.007$ & $0.965 \pm 0.006$ \\
\hline 1 & $0.894 \pm 0.007$ & $0.95 \pm 0.01$ & $0.98 \pm 0.01$ & $0.973 \pm 0.008$ \\
\hline 2 & $0.852 \pm 0.009$ & $0.955 \pm 0.004$ & $0.985 \pm 0.003$ & $0.990 \pm 0.002$ \\
\hline
\end{tabular}

Table 2. Fidelity of quantum state with $\mathrm{N}=8$, backend Toronto, and $\mathrm{t}=1$.

Finally, we consider the case where $N=16$ in table 3 . Here, the behaviour is similar to the previous case up to graph $G_{5}$, meaning that higher graph connectivity and larger $m$ will result in higher fidelity. However, graphs $G_{6}, G_{7}$ and $G_{8}$ even though are highly connected and have relatively low depth, present lower fidelity. This is due to the fact that the probability distribution of the dynamic of the walk on these structures is highly concentrated in a small number for vertices, which means they are more susceptible to noise introduced by NISQ computers. Nonetheless, the increase of $m$ still has a positive impact on the fidelity of these circuits.

\begin{tabular}{|l|l|l|l|l|l|l|l|l|}
\hline $\mathrm{m} \backslash G$ & G1 & G2 & G3 & G4 & G5 & G6 & G7 & G8 \\
\hline 0 & $0.47 \pm 0.03$ & $0.61 \pm 0.02$ & $0.78 \pm 0.02$ & $0.86 \pm 0.01$ & $0.86 \pm 0.01$ & $0.70 \pm 0.04$ & $0.54 \pm 0.03$ & $0.49 \pm 0.04$ \\
\hline 1 & $0.50 \pm 0.03$ & $0.63 \pm 0.03$ & $0.79 \pm 0.03$ & $0.87 \pm 0.02$ & $0.85 \pm 0.03$ & $0.70 \pm 0.03$ & $0.55 \pm 0.05$ & $0.50 \pm 0.04$ \\
\hline 2 & $0.55 \pm 0.03$ & $0.71 \pm 0.03$ & $0.83 \pm 0.02$ & $0.90 \pm 0.01$ & $0.89 \pm 0.02$ & $0.75 \pm 0.02$ & $0.62 \pm 0.04$ & $0.59 \pm 0.06$ \\
\hline 3 & $0.60 \pm 0.03$ & $0.70 \pm 0.02$ & $0.85 \pm 0.01$ & $0.92 \pm 0.01$ & $0.91 \pm 0.01$ & $0.80 \pm 0.03$ & $0.71 \pm 0.04$ & $0.69 \pm 0.04$ \\
\hline
\end{tabular}

Table 3. Fidelity of quantum state with $\mathrm{N}=16$, backend Toronto, and $\mathrm{t}=1$.

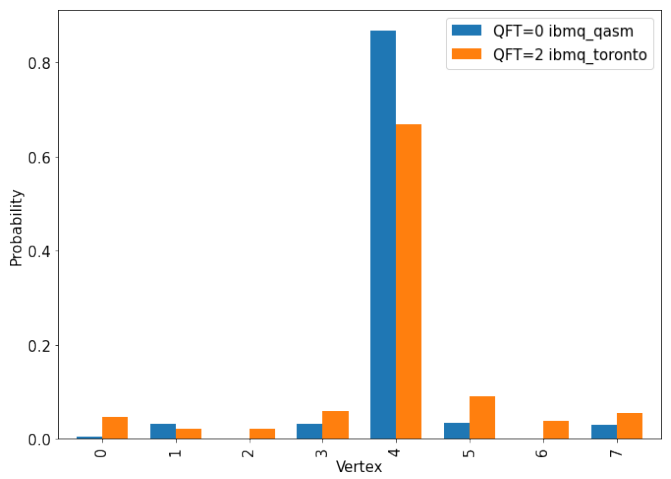

(a) $v_{1}=[0,1,0,0,0,0,0,1]^{T}$

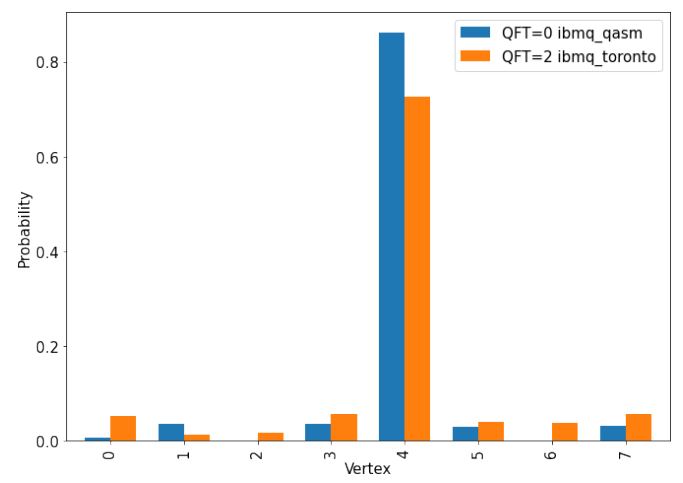

(b) $v_{2}=[0,1,1,0,1,0,1,1]^{T}$

Figure 5. Pretty good state transfer for different circulant graphs defined by $v$.

We implemented pretty good state transfer (PGST) which is a transport phenomon that occurs in quantum walks when the initial state is restricted to a single excitation space. PGST occurs between vertex $i$ and $j$ if, for any $\epsilon>0$, there exists a time $\tau_{\epsilon}$ such that

$$
\left|U\left(\tau_{\epsilon}\right)_{i j}\right|>1-\epsilon .
$$

It was proved by Pal et al. [Pal and Bhattacharjya 2017] that PGST occurs in some classes of circulant graphs at $\tau_{\epsilon}=2 \pi n$, where $n$ is a positive integer. One of those classes is cycles with $2^{k}$ vertices $\left(C_{2^{k}}\right)$, where $k \geq 3$, and the other is the union $C_{2^{k}} \cup G\left(2^{k}\right)$, with $G\left(2^{k}\right)$ being circulant graphs with connection set given by gcd-sets of $\mathbb{Z}_{2^{k}}$. We chose one graph of each class, $v_{1}=[0,1,0,0,0,0,0,1]^{T}$ and $v_{2}=[0,1,1,0,1,0,1,1]^{T}$, and we 
obtained a fidelity of $0.93 \pm 0.01$ for $v_{1}$ and $0.906 \pm 0.009$ for $v_{2}$. Figure 5 shows the comparison between the simulated model in blue and the experimental result in orange.

\section{Conlusion and Remarks}

We presented a general framework of implementing the CTQW for an arbitrary circulant graph of any size. Previous work by [Qiang et al. 2016] implemented the CTQW through the QFT for a complete graph of small size, however our method takes advantage of the Qiskit package making it possible to create a circuit for absolutely any circulant graph while making use of the AQFT in order to reduce circuit depth. Contrasting our statistical analysis to the work of [Acasiete et al. 2020], we offer a more accurate overview of the results of running a quantum walk on a superconducting NISQ device, since we calculated the average fidelity of several instances instead of choosing the best case. Finally, we presented two cases of PGST were we also explored the effects of the AQFT.

\section{References}

Acasiete, F., Agostini, F. P., Moqadam, J. K., and Portugal, R. (2020). Implementation of quantum walks on IBM quantum computers. Quantum Information Processing, 19(12).

Ambainis, A. (2008). Quantum Algorithm for Element Distinctness, pages 686-689. Springer US, Boston, MA.

Balu, R., Castillo, D., and Siopsis, G. (2018). Physical realization of topological quantum walks on IBM-q and beyond. Quantum Science and Technology, 3(3):035001.

Bullock, S. S. and Markov, I. L. (2004). Smaller circuits for arbitrary n-qubit diagonal computations. Quant. Info. and Comp., 4(27).

Chakraborty, S., Luh, K., and Roland, J. (2020). How fast do quantum walks mix? Phys. Rev. Lett., 124:050501.

Childs, A. M. and Goldstone, J. (2004). Spatial search by quantum walk. Physical Review A, 70(2):022314.

Coutinho, G. (2014). Quantum State Transfer in Graphs. PhD thesis, University of Waterloo.

Farhi, E. and Gutmann, S. (1998). Quantum computation and decision trees. Physical Review A, 58:915-928.

Pal, H. and Bhattacharjya, B. (2017). Pretty good state transfer on circulant graphs. The Electronic Journal of Combinatorics, 24.

Qiang, X., Loke, T., Montanaro, A., Aungskunsiri, K., Zhou, X., O’Brien, J. L., Wang, J. B., and Matthews, J. C. F. (2016). Efficient quantum walk on a quantum processor. Nature Communications, 7:11511.

Shakeel, A. (2020). Efficient and scalable quantum walk algorithms via the quantum Fourier transform. Quantum Information Processing, 19(9):323.

Shende, V., Bullock, S., and Markov, I. (2006). Synthesis of quantum-logic circuits. IEEE Transactions on Computer-Aided Design of Integrated Circuits and Systems, 25(6):1000-1010. 